\title{
CORRIGENDUM \\ Effect of storage conditions on losses and crop utilization of nitrogen from solid cattle manure - CORRIGENDUM
}

\author{
G. M. SHAH, G. A. SHAH, J. C. J. GROOT, O. OENEMA, A. S. RAZA and E. A. LANTINGA
}

In the above-mentioned paper (Shah et al. 2015), the author regrets to announce that an incorrect version of Table 3 and Fig. 3 were given in the published article.

The correct versions are supplied below.

Table 3. Summary of nitrogen $(\mathrm{N})$ balance for solid cattle manure stored under different conditions $(\mathrm{n}=3)$

\begin{tabular}{|c|c|c|c|c|c|c|c|c|c|c|}
\hline \multirow[b]{2}{*}{ Treatment } & Initial & Final & Difference & $\begin{array}{l}\text { Total } \mathrm{NH}_{3-} \\
\mathrm{N} \text { emission }\end{array}$ & $\begin{array}{l}\text { Total } \mathrm{N}_{2} \mathrm{O}- \\
\mathrm{N} \text { emission }\end{array}$ & $\begin{array}{l}\text { Total N } \\
\text { leached }\end{array}$ & \multicolumn{2}{|c|}{ Measured $\mathrm{N}$ losses } & \multicolumn{2}{|c|}{$\begin{array}{l}\text { Unaccounted N } \\
\text { losses }\end{array}$} \\
\hline & \multicolumn{6}{|c|}{ (kg/heap) } & $\begin{array}{l}(\mathrm{kg} / \\
\text { heap) }\end{array}$ & $\begin{array}{l}\text { (\% of total } \\
\mathrm{N} \text { losses })\end{array}$ & $\begin{array}{l}(\mathrm{kg} / \\
\text { heap) }\end{array}$ & $\begin{array}{l}(\% \text { of total } \\
\mathrm{N} \text { losses })\end{array}$ \\
\hline Roofed & $63 \cdot 1$ & $55 \cdot 5$ & $8 \pm 1 \cdot 9(12)$ & 0.67 & $0 \cdot 32$ & $1 \cdot 2$ & $2 \cdot 2^{*}$ & 28 & $5 \cdot 4+$ & 72 \\
\hline Stockpiled & $65 \cdot 3$ & $51 \cdot 8$ & $14 \pm 2 \cdot 5(21)$ & $0 \cdot 26$ & 0.07 & $3 \cdot 2$ & $3 \cdot 5$ & 26 & $10 \cdot 0$ & 74 \\
\hline Turned & $74 \cdot 7$ & $50 \cdot 3$ & $24 \pm 4 \cdot 0(33)$ & $0 \cdot 77$ & $0 \cdot 14$ & $3 \cdot 8$ & $4 \cdot 7$ & 19 & $19 \cdot 7$ & 81 \\
\hline Covered & $67 \cdot 7$ & $63 \cdot 6$ & $4 \pm 1 \cdot 2(6)$ & N/A & $\mathrm{N} / \mathrm{A}$ & $1 \cdot 3$ & $1 \cdot 3$ & 32 & $2 \cdot 8$ & 68 \\
\hline
\end{tabular}

N/A, not applicable.

Values in the parenthesis are percentages of the initial amount.

* Measured $\mathrm{N}$ losses $(\mathrm{kg} /$ heap $)=\left(\mathrm{NH}_{3}-\mathrm{N}+\mathrm{N}_{2} \mathrm{O}-\mathrm{N}+\right.$ total $\mathrm{N}$ leached $)$.

+ Unaccounted $\mathrm{N}$ losses $(\mathrm{kg} /$ heap $)=($ initial total $\mathrm{N}-$ final total $\mathrm{N})-$ measured $\mathrm{N}$ losses.

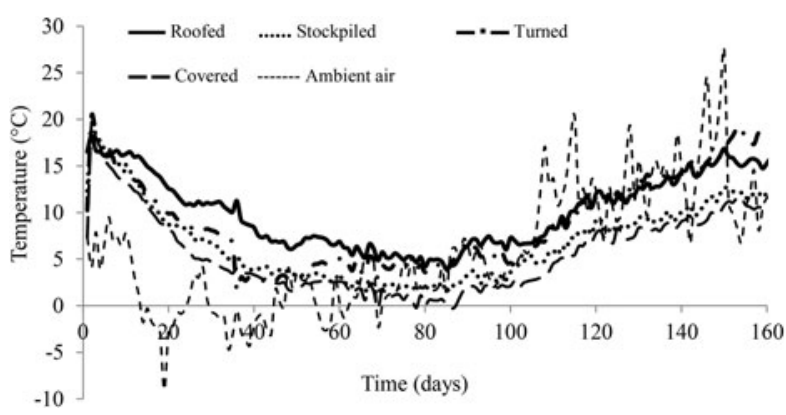

Fig. 3. Average temperature at the depth of $1 \mathrm{~m}$ from the surface of the manure heaps during the storage period.

\section{REFERENCE}

Shah, G. M., Shah, G. A., Groot, J. C. J., Oenema, O., Raza, A. S. \& Lantinga, E. A. (2015). Effect of storage conditions on losses and crop utilization of nitrogen from solid cattle manure. The Journal of Agricultural Science, available on CJO2015. doi:10.1017/S0021859614001348. 